\title{
DEBATE
}

\section{Genital ulcers, stigma, HIV, and STI control in sub-Saharan Africa}

\section{N O'Farrell}

Sex Transm Infect 2002;78:143-146

HIV associated stigma is still prevalent throughout Africa despite the spread of the epidemic. Stigma is also attached to sexually transmitted infections (STIs). Despite the importance of STIs, particularly genital ulceration in facilitating heterosexual HIV transmission, policymakers continue to focus mainly on other priorities. It would appear that this lack of public health initiative in tackling genital ulcers is itself an example of stigmatisation. Possible explanations for this include geographical variation in the data and a perception that genital ulcers are not a topic that can be discussed freely and openly. HIV policymakers in countries worst affected by HIV in Africa should examine their own opinions for bias when determining public health priorities for HIV prevention. The importance of genital ulcers should be reassessed and publicised.

Correspondence to: Pasteur Suite, Ealing Hospital, Uxbridge Road, London UB $13 \mathrm{HW}$, UK; ofarrell@postmaster.co.uk

Accepted for publication 13 December 2001
$\mathrm{F}$ ew individuals with HIV in Africa have been open about their diagnosis. Despite the spread of HIV, the stigma attached to HIV disclosure has barely diminished over the years. Stigma is also attached to sexually transmitted infections (STIs). A significant proportion of patients with STIs in Africa either delay in seeking health care or prefer to see general practitioners or traditional healers in an anonymous setting rather than go to a designated STI clinic, despite the availability of both better treatment and clinical expertise in the latter.

STI related stigma is brought about by many factors including feelings of guilt, low self esteem, uncleanliness and unworthiness, possibly through having paid for sex. In parallel with these negative perceptions at the individual level, at the public health level it could be said that resource allocation and STI expertise in developing countries have also been victims of stigma. Evidence linking the importance of STIs in facilitating HIV transmission first identified 15 years ago $^{1}$ has not been matched by responses at policymaking level to improve STI services. Few policymakers find out for themselves how members of a community put themselves at risk of STIs and HIV. Indeed, it almost seems that policymakers view STI services as a suitable case for stigmatisation.

This lack of focus on STI issues has meant that genital ulceration, a risk factor consistently associated with enhancing heterosexual HIV transmission, ${ }^{2}$ has been unduly neglected. This article discusses reasons why genital ulceration has been largely ignored and puts forward a case for specific interventions to limit genital ulcers as part of a strategy for overall HIV prevention and control.

\section{WHY HAVE GENITAL ULCERS BEEN IGNORED?}

Genital ulceration has probably been ignored for two main reasons-disparities in the epidemiological evidence and political correctness or bias.

\section{The epidemiological evidence}

In areas where the HIV epidemic is worst, prevalence of genital ulcers is very high. ${ }^{3}$ The genital ulcer cofactor effect for HIV transmission has been estimated at 10-50 for male to female and 50-300 for female to male per sexual contact among female sex workers and their clients. ${ }^{4}$ Furthermore, simulation modelling scenarios of the transmission dynamics of HIV infection estimated that genital ulcers were responsible for $83 \%$ of HIV infections in low cofactor scenarios and $97 \%$ in a high cofactor scenario for the first 10 years of the epidemic in Uganda.

Despite the evidence that genital ulcers are as major risk factor for the transmission of HIV both from male to female and vice versa, doubters still remain. The heterosexual HIV epidemic is characterised by marked heterogeneity so that risk factors identified in one area may be much less important elsewhere. For example, the epidemiological evidence for genital ulcers in enhancing HIV transmission varies between populations: odds ratios and risk ratios ranging from 2-13.5 were identified in a systematic review. ${ }^{6}$ STI epidemiology also varies considerably between geographical locations and with time and stage of the HIV epidemic. The need for a country specific approach to STI control in which local disease patterns are identified and targeted is therefore crucial. $^{7}$ For example, whereas chancroid and syphilis were thought to be common in developing country settings, genital herpes is now the most common cause of genital ulcer in many African countries. ${ }^{8}$ This emergence of genital herpes requires that STI management algorithms for genital ulcer be updated and include education and discussion at the individual level. However, many herpetic ulcers go undiagnosed because of delays in seeking health care. Educating patients to recognise minor recurrent herpetic sores could have a significant impact on the population attributable risk of HIV transmission due to herpetic infection.

Achieving acceptance of the role of genital ulcers as a risk factor for HIV has also been hindered by other factors, including variation in what is understood by the term genital ulcer and 
poor diagnostic tests. The accepted definition of a genital ulcer is a lesion on the surface of the skin caused by superficial loss of tissue usually with inflammation. ${ }^{9}$ The biological mechanism whereby HIV transmission is facilitated through genital ulcers is thought to be via the ulcer providing a portal of entry and exit for the virus. If this is accepted, any breaks in genital mucosa should be considered as a potential anatomical site for HIV transmission and the concept of genital ulceration as a risk factor for HIV expanded to include all such breaks, whether or not they are sexually transmitted ulcers or simple mucosal tears. Tests for the STI causes of genital ulcers are not always available or accurate: culture methods for chancroid and herpes may have a sensitivity of less than $50 \%$. Although polymerase chain reaction tests are now becoming more accessible than before, their use in Africa is still minimal. Furthermore, many ulcers still remain undiagnosed despite using this technology. ${ }^{10}$

The lack of importance currently attached to clinical examination to confirm the presence of ulcers is also a cause for concern. In a simulated patient assessment of the quality of STI management in a rural area of KwaZulu, South Africa, only $19 \%$ were offered physical examination. ${ }^{11}$ In men, genital ulcers should be obvious on clinical examination, assuming that the foreskin is retractile. In women the diagnosis of external ulcers should be straightforward but cervical lesions require a speculum examination and may be difficult to differentiate from cervical ectopy. Promotion of clinical examination so that areas of mucosal discontinuity can be identified at the individual level would seem to offer more benefits to men than women. Unfortunately, it appears that clinical examination of the genitalia has fallen by the wayside following the recent emphasis on STIs as a public health issue. This is regrettable, as it is these individuals with STIs, many of whom are STI repeaters, HIV positive, and attend clinics, who should be classified as a key core group at high risk of HIV transmission and targeted for HIV prevention interventions.

\section{Political bias/correctness}

Most issues around HIV, particularly funding, have always had a political agenda. Some HIV prevention priorities are deemed to be politically correct whereas other worthy interventions may be ignored because they do not fit in with the wider objectives of aid organisations. For example, while many multinational agencies have focused on poverty reduction to impact on AIDS, this policy fails to target many of those at very high risk of infection. Also, poor male genital hygiene, a risk factor that could be important in driving the HIV epidemic in Africa, ${ }^{12}$ has probably been ignored because of the possibility that HIV might be viewed or promoted as a disease of the unclean, thereby leading to further stigmatisation.

Despite the importance of targeting high risk groups in combatting the HIV epidemic, most interventions have tended to focus on either comparatively small groups who can be accessed by project work directly or the population as a whole, many of whom are at low risk of HIV. The notion that every sexually active individual is at risk of HIV probably reflects the early campaigns of gay activists who did not want to be seen as the vectors of disease. Even now targeting high risk groups is frowned upon because they may be blamed for spreading the epidemic.

A further factor in Africa is the issue of political leadership. Currently, all African heads of state are men. Many of these male dominated governments appear to have a puritanical view of sex, possibly as a consequence of missionary schooling, with the result that sexual matters are not discussed openly. In reality, the sexual behaviour of these male government leaders may be very different from that expected from their upbringing and not something they are prepared to discuss in public. Rather than address the question of STIs, it would seem easier for them to ignore such matters completely rather than become tainted through association and risk having double standards exposed.

Another reason for the apparent lack of emphasis on STI control for HIV prevention has been the assumption that syndromic management is the best strategy available. However, it could be said that STI syndromic management policies have failed to contain the spread of HIV. In its purest form syndromic management entails treating STIs by simple methods for the most likely causes of a syndrome, usually with at least two antibiotics without laboratory tests. While this approach has been used for a long time by many medical practitioners, it is perhaps too simplistic and has been open to misinterpretation. For example, while syndromic management protocols have simplified drug treatments at the primary healthcare level, the other essential components of STI case management including health education, partner notification, and condom promotion are often missed out. ${ }^{11}$ Increased numbers of trained, motivated, and well supervised primary care personnel, capable of managing all aspects of STIs, are required so that comprehensive STI case management is delivered at the first point of contact with a health agency.

Although sex issues are to the forefront of the political HIV agenda, there have been few interventions targeting men who are generally regarded as the perpetrators of the HIV epidemic rather than women who are the victims. Interventions to target men, particularly those with genital ulcers, have therefore not been popular despite the clear need that they should be targeted equally if not more than women. ${ }^{13}$

\section{IMPLICATIONS \\ Stigma}

The perception of stigma is an important barrier to limiting the spread of HIV and STI in sub-Saharan Africa. Although communities perceive stigma in different ways, individuals with STIs often anticipate feelings of rejection and shame that lead to long delays in seeking health care. ${ }^{14}{ }^{15}$ Part of this delay may be attributed to a preference for traditional healers who are still viewed by many as having the best treatment for STIs even though the efficacy of such treatments remains unproved. ${ }^{16}$ National AIDS prevention programmes must now consider how this STI associated stigma can be reduced in their communities. Specific country and culture specific investigations are needed to identify the origins of stigma and how best to minimise its consequences.

One area that is poorly described in the literature is the interaction between stigma and sex work. Early on in the HIV epidemic a considerable amount of HIV/STI research work in Africa was undertaken in female sex workers. This work was well publicised and it is likely that the belief that HIV was related to commercial sex work was reinforced in both sexes through these studies. At the population level, the vast majority of women in Africa with HIV are infected by their only sexual partner and it is understandable how these women may feel ashamed when identified as HIV positive if they believe that female sex workers are the major HIV risk group. Although successful sex work interventions have been implemented in Asia, in many parts of Africa sex work is informal and not controlled. To enforce a $100 \%$ condom use policy, so successful in Thailand, would therefore not be realistic in Africa. What is needed is the recognition and acceptance by men that they, rather than women, are the main source vectors of infection and that the high per act HIV transmission rates from core groups are driving the epidemic. ${ }^{13}$

\section{Simplification or not?}

If the importance of mucosal breaks in the skin with subsequent inflammation is accepted as a crucial factor for female to male HIV transmission, HIV control programmes should become easier to devise and implement. However, explaining HIV transmission in simple biological terms may 
be unpopular as a concept because it is not politically correct. Over the past 20 years, HIV related issues have grown almost into an industry. Any move to simplify matters might be resisted by agencies who would see this simplification strategy as a threat to their existence.

Throughout sub-Saharan Africa condom use still appears to be low. Moreover, sexual intercourse in the presence of genital ulcer is common and a practice that still continues despite the emergence of the HIV epidemic. ${ }^{17-19}$ A renewed focus on the biological determinants of HIV transmission could offer effective alternative strategies to those currently promoted.

\section{CONCLUSIONS}

One of the most dramatic success stories in HIV prevention has been in Thailand where the $100 \%$ condom use campaign and an increased number of quality STI clinics brought about a significant reduction in the prevalence of STIs and HIV. ${ }^{20}$ Despite the incidence of most important STIs in Africa being double that in Asia, ${ }^{21}$ there are still very few specialist clinics in Africa. The few STI specialists that there are in Africa tend to work in the private sector and have limited interest in public health control of STIs. Also, research teams working on STI projects in Africa are usually composed of microbiologists or public health physicians who, with a few notable exceptions, rarely manage STI patients. The dearth of specialist STI input and advocacy has, almost by default, led to significant investment in large scale public health interventions such as the mass treatment project for STIs in Rakai, Uganda, in which population coverage was limited and HIV incidence was not reduced. ${ }^{10}$ In some ways this promotion of STIs as a public health problem that can be dealt with by "a magic bullet" approach has reinforced the perception that STIs are still unclean and can be dealt with "out of sight" requiring neither clinical examination nor health education. Furthermore, the move to decentralise STI services to primary health care advocated by the World Health Organization since the 1970s has not made sufficient impact in reducing STI enhanced HIV transmission and neither has the move to integrate STI services with family planning clinics resulted in a demonstrated reduction in STIs. ${ }^{22}$ The adoption of the public health approach to STI control by UN agencies ${ }^{23}$ has ultimately been at the expense of promoting quality STI services in non-judgmental non-stigmatising settings for individuals at high risk of HIV who are driving the epidemic. Clearly, health ministries must now consider STI control as a special case for resource allocation if the deficiencies that have accumulated over the years are to be eradicated. An increase in designated STI clinics with trained well motivated specialist STI healthcare workers would not only raise the profile of STIs but also have tremendous potential to reduce STI related stigma. ${ }^{24}$

The reluctance to accept the importance of genital ulceration in some ways mirrors the importance of lack of circumcision as a risk factor for HIV, a finding that is only now gaining acceptance. Also, the reticence of policymakers to accept the importance of biological risk factors for HIV may only reflect embarrassment in admitting that previous priorities were misdirected. ${ }^{25}$ Clearly, innovative treatment strategies to tackle genital ulcers are necessary and further research work must be done to determine the most cost effective approaches. Mass treatment for syphilis with a single dose of benzathine penicillin should be considered, if not at the population level, at least on an opportunistic basis for all STI cases presenting to a healthcare facility in areas where syphilis is a significant problem. It is probably too late to consider mass treatment for chancroid as the prevalence of this condition has now diminished considerably. Genital herpes requires a different approach. Selective mass treatment for core groups could be considered but education and counselling interventions are also essential. In donovanosis endemic countries, eradication is a viable proposition. Advice about how best to deal with non-STI induced breaks of skin due to trauma or poor hygiene would be the same as for any ulcer-that is, to abstain from unprotected sex until complete healing had been achieved.

Although non-ulcerative STIs enhance HIV transmission, ${ }^{26}$ the majority of HIV infections occur through mucosal disruption either on the penis or the cervix. However, rather than focusing on this biological model, health agencies have instead advocated a simple syndromic approach to managing STIs that has often involved considerable overtreatment with scarce antibiotics. Clearly, this approach has been inadequate. While syndromic management may be cost effective for certain syndromes in some areas, this strategy has been rejected in many countries where it should have been effective if combined with a comprehensive case management approach. If national STI/HIV control programmes were to develop a more country specific strategy, local problems could be targeted and guidelines developed that might be more acceptable than some generic versions currently in use.

Currently the UN and other multinational agencies are becoming increasingly involved with complex economic and human rights issues, including the subordination of women, which while important, fail to address many of the issues driving the rapid spread of HIV in those countries worst affected by the epidemic. This sentiment was reinforced by both the recent 40 point Abuja declaration on HIV/AIDS made on behalf of the heads of government of the Organisation of African Unity and the 103 point UN declaration of commitment on HIV/AIDS in which the importance of STIs is not mentioned once. ${ }^{27}{ }^{28}$ The challenge remains not only to get the funding agencies and health ministries interested in a back to basics approach to STI control with a focus on genital ulcers in countries worst affected by the epidemic, but also to reduce any STI related stigma they may harbour against STI control programmes.

\section{REFERENCES}

1 Kreiss JK. AIDS virus infection in Nairobi prostitutes: spread of the epidemic to East Africa. N Engl J Med 1986:314:414-18.

2 Dickerson MC, Johnston J, Delea TE, et al. The causal role for genital ulcer disease as a risk factor for transmission of human immunodeficiency virus. Sex Transm Dis 1996;23:429-40.

$3 \mathrm{O}^{\prime}$ Farrell $\mathbf{N}$. The need for targeted interventions against genital ulcers in countries worst affected by HIV in Africa. Bull World Health Organ $2001 ; 79: 569-77$

4 Hayes RJ, Schulz KF, Plummer FA. The cofactor effect of genital ulcers on the per-exposure risk of HIV transmission. J Trop Med Hyg 1995; $98: 1-8$.

5 Robinson NJ, Mulder DW, Auvert B, et al. Proportion of HIV infections attributable to other sexually transmitted diseases in a rural Ugandan population: simulation model estimates. Int J Epidemiol 1997;26:180-9.

6 Fleming DT, Wasserheit JN. From epidemiological synergy to public health policy and practice: the contribution of other sexually transmitted diseases to sexual transmission of HIV infection. Sex Transm Inf 1999:75:3-17.

7 O'Farrell N. The commonwealth and HIV: the need for a country-specific approach. In: HIV/AIDS in the Commonwealth 2000/2001 (2000). Kensington Publications for the Commonwealth secretariat.

8 O'Farrell N. Increasing prevalence of genital herpes in developing countries: implications for heterosexual HIV transmission and STI control programmes. Sex Transm Inf 1999;75: 377-84.

9 Stedman's medical dictionary. 26th ed. Philadelphia: Williams \& Wilkins, 1995.

10 Wawer MJ, Sewankambo NK, Serwadda D, et al. Control of sexually transmitted diseases for AIDS prevention in Uganda: a randomised community trial. Lancet 1999;353:525-35.

11 Harrison A, Wilkinson D, Lurie M, et al. Improving quality of sexually transmitted disease case management in South Africa. AIDS 1998;12 2329-2335

12 O'Farrell N. Soap and water prophylaxis for limiting genital ulcer disease and HIV-1 infection in men in sub-Saharan Africa. Genitourin Med 1993;69:297-300

13 O'Farrell N. Enhanced efficiency of female-to-male HIV transmission in core groups in developing countries: the need to target men. Sex Transm Dis 2001;28:84-91.

14 Moss W, Bentley M, Maman S, et al. Foundations for effective strategies to control sexually transmitted infections: voices from rural Kenya. AIDS Care 1999;11:95-113.

15 Nuwaha F, Faxelid E, Neema S, et al. Lay people's perceptions of sexually transmitted infections in Uganda. Int J STD AIDS 1999;10:709-17. 
16 Goldin CS. Stigmatisation and AIDS: critical issues in public health. Soc Sci Med 1994;39: 1359-66.

17 O'Farrell N, Hoosen AA, Coetzee K, et al. Sexual behaviour in Zulu men and women with genital ulcer disease. Genitourin Med 1992:68:245-8

18 Morgan D, Mahe C, Okango JM, et al. Genital ulceration in rural Uganda: sexual activity, treatment-seeking behaviour, and the implications for HIV control. Sex Transm Dis 2001;28:431-6.

19 Fonck K, Mwai C, Rakwar J, et al. Healthcare seeking behaviour and sexual behavior of patients with sexually transmitted diseases in Nairobi, Kenya. Sex Transm Dis 2001;28:367-71.

20 Hanenberg RS, Rojanapithayakorn W, Kunasol P, et al. Impact of Thailand's HIV-control programme as indicated by the decline of sexually transmitted diseases. Lancet 1994;344:243-5.

21 Gerbase AC, Rowley JT, Heymann DH, et al. Global prevalence and incidence estimates of selected curable STDs. Sex Transm Inf 1998;74(Suppl 1): S12-16

22 World Health Organization. Integrating STI management into family planning services. What are the benefits? WHO/RHR/99.10. Geneva: $\mathrm{WHO}$.
23 UNAIDS. The public health approach to STD control: UNAIDS Technical Update 1998.

24 Scoular A, Duncan B, Hart G. "That sort of place . . . where filthy men go ....": a qualitative study of women's perceptions of genitourinary medicine services. Sex Transm Inf 2001;77:340-3.

25 Caldwell JC, Caldwell P. The neglect of an epidemiological explanation for the distribution of HIV/AIDS in sub-Saharan Africa: exploring the male circumcision hypothesis. Health Transition Review 1994;4(Suppl):23-46.

26 Laga M Manoka A Kivuvu M et al. Non-ulcerative sexually transmitted diseases as risk factors for HIV-I infection in women: results from a cohort study. AIDS 1993;7:95-10

27 http://www.oau-oua.org/afsummit/ HIV-AIDS\%20TB\%ORID\%20ABUJA\%20declaration.htm

28 United Nations General Assembly. 26th Special Session, 27 June 2001, New York. Declaration of Commitment on HIV/AIDS. Global Crisis- Global Action. www.hivnet.ch:8000/africa/af-aids/viewR? 1113

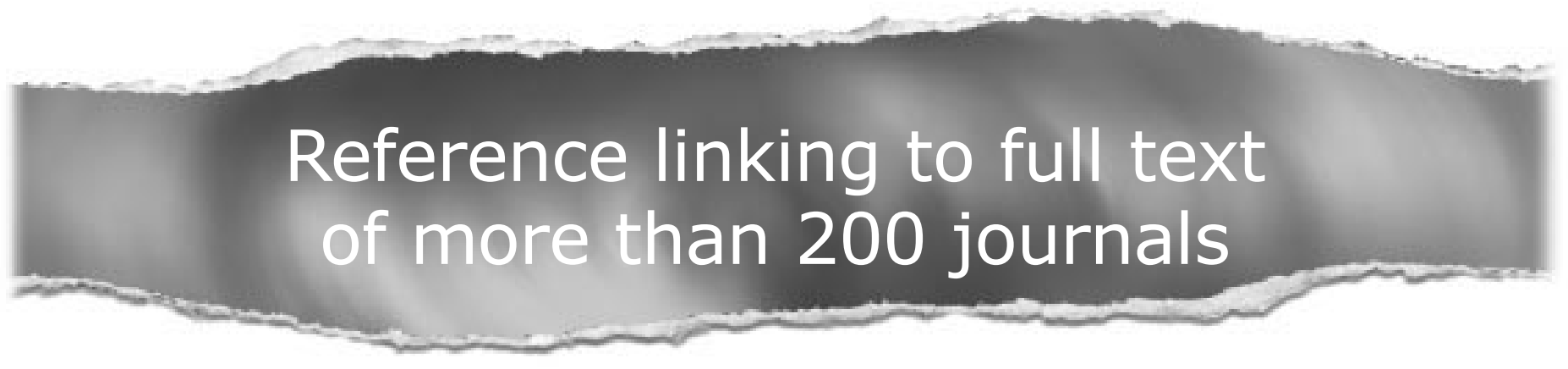

\section{Toll free links}

You can access the FULL TEXT of articles cited in Sexually Transmitted Infections online if the citation is to one of the more than 200 journals hosted by HighWire (http://highwire.stanford.edu) without a subscription to that journal. There are also direct links from references to the Medline abstract for other titles.

www.sextransinf.com 